Rongping Wang et al., Identifying the best chalcogenide glass compositions for the application in mid-infrared waveguides. Proc. SPIE 9444, International Seminar on Photonics, Optics, and Its Applications (ISPhOA 2014), 944406 (January 9, 2015).

Invited Paper Copyright 2015 Society of Photo Optical Instrumentation Engineers. One print or electronic copy may be made for personal use only. Systematic reproduction and distribution, duplication of any material in this paper for a fee or for commercial purposes, or modification of the content of the paper are prohibited. http://doi.org/

\title{
Identifying the best chalcogenide glass compositions for the application in mid-infrared waveguides
}

\author{
Rongping Wang, Xueqiong Su, Douglas Bulla, Ting Wang, Xin Gai, Zhiyong Yang, Steve Madden \\ and Barry Luther-Davies
}

\author{
Centre for Ultrahigh Bandwidth Devices for Optical Systems (CUDOS), Laser Physics Centre, \\ Research School of Physics and Engineering, Australian National University, Canberra, ACT 0200, \\ Australia
}

\begin{abstract}
We prepared numbers of GeAsSe glasses and investigated their thermal stability and optical properties in order to search the best glasses with relatively high glass transition temperature $T_{\mathrm{g}}$, strong structural stability, low optical loss and high optical nonlinearity. Through our systematical measurements, we concluded that the glasses with a mean coordination number around 2.45-2.5 are the best for the applications in photonics with $\mathrm{T}_{\mathrm{g}}$ of $450 \mathrm{~K}$, low optical loss of $0.2 \mathrm{~dB} / \mathrm{cm}$, high optical nonlinearity $7.5 \times 10^{-14} \mathrm{~cm}^{2} / \mathrm{W}$ and less photosensitivity.
\end{abstract}

Keywords: Chalcogenide glasses, photonics

\section{Introduction}

Chalcogenide glasses are the materials containing one or more of the chalcogen elements sulphur, selenium and tellurium from group VI of the periodic table that are covalently bonded to network formers such as $\mathrm{Si}, \mathrm{P}, \mathrm{Ge}, \mathrm{As}$, Ge or $\mathrm{Sb}$. They are an important class of photonics materials with unique properties including low phonon energy, ultrafast response time, and high optical nonlinearity ${ }^{1,2}$. This makes them good options of the material system to be used in optical devices. For example, binary glasses such as $\mathrm{As}_{2} \mathrm{~S}_{3}$ and $\mathrm{As}_{2} \mathrm{Se}_{3}$ have been used to produce waveguide devices with high performance, as well as draw into optical fibres for the applications in mid infrared region ${ }^{3-6}$. However, properties such as the nonlinearity and glass transition temperatures, $\mathrm{T}_{\mathrm{g}}$, of these binary glasses can be further improved. Generally it is expected that the addition of Ge with 4-fold bond coordination compared with As (3-fold) and S/Se (2fold) produces a more 3-dimensional glass network and this increases $T_{g}$. These trends have motivated research into ternary (also quaternary) compounds and recently Ge-As-Se-based glasses with an optical nonlinearity that is hundreds or thousands time than binary glasses have been demonstrated ${ }^{7-9}$. Moreover, it has been well known that the properties of the ternary glasses usually can be tuned in a widely compositional range. Therefore to search the best glasses with proper compositions is essential for the applications in photonics. In the present paper, we systematically investigated the glassforming properties, thermal stability and optical properties of the Ge-As-Se glasses with an aim to identify the best GeAs-Se compositions for the applications in photonics.

\section{Experiments}

Wide range of Ge-As-Se bulk glasses were prepared from high purity elements using the conventional melt quenching technique. High purity (5N) Germanium, Arsenic and Selenium metals used as starting materials were weighed inside a dry nitrogen glove box and loaded into a pre-cleaned quartz ampoule. The loaded ampoule was dried under vacuum $\left(10^{-6}\right.$ Torr) at $110^{\circ} \mathrm{C}$ for 4 hours to remove surface moisture from the raw materials. The ampoule was then sealed under vacuum using an oxygen-hydrogen torch, and introduced into a rocking furnace to melt the contents at $900^{\circ} \mathrm{C}$ for a period not less than 30 hours. The temperature was then reduced at a rate of $3^{\circ} \mathrm{C}$ per minute until it reached $600-700^{\circ} \mathrm{C}$, depending on the exact glass composition. The ampoule was subsequently removed from the rocking furnace at this temperature before being air quenched. The resulting glass boule was annealed at a temperature $30^{\circ} \mathrm{C}$ below $\mathrm{T}_{\mathrm{g}}$, then slowly cooled to room temperature. Following the annealing process, the glass boules were sectioned to form discs of 10

International Seminar on Photonics, Optics, and Its Applications (ISPhOA 2014), edited by Aulia Nasution, Proc. of SPIE Vol. 9444, 944406 - @ 2015 SPIE - CCC code: 0277-786X/15/\$18 - doi: 10.1117/12.2074815 
mm diameter and approx $2 \mathrm{~mm}$ thick. The discs had their opposite surfaces ground plane parallel and then polished to optical quality.

An infrared microscope with a detector sensitive to a wavelength of $2.2 \mu \mathrm{m}$ was used to search for impurities present in the glasses, and the results revealed the absence of any observable bubbles for all the glasses. The chemical compositions of both the bulk glass were analysed by energy dispersive x-ray analysis (EDX) installed on a s canning electron microscope using commercial $\mathrm{Ge}_{33} \mathrm{As}_{12} \mathrm{Se}_{55}$ as reference standard. The high quality bulk glasses were also employed as the sources to prepare Ge-As-Se thin films under vacuum using a thermal evaporator. The amorphicity of the bulk glasses and films were checked by $\mathrm{x}$-ray diffraction (XRD) using a conventional x-ray diffractometer in a $2 \theta$ scan mode. About $15 \mathrm{mg}$ bulk glass powder was sealed into an aluminium pan for $\mathrm{T}_{\mathrm{g}}$, activation energy of glass transition and fragility measurements using a differential scanning calorimeter (Mettler-Toledo, DSC 1). The refractive indices of bulk Ge-Sb-Se glasses were measured using a M etricon Model 2010 prism coupler. The absorption spectra were recorded using a Varian Cary 5000, UV-Vis-NIR spectrophotometer in the wavelength range from 400 to $1500 \mathrm{~nm}$. The optical gap was estimated at $\sim 10^{3} \mathrm{~cm}^{-1}$. The refractive index and optical band gap of the films were measured by FilmTek 4000.The nonlinearities were measured using $1-2 \mathrm{~mm}$ thick samples via the $\mathrm{z}$-scan technique. The nonlinear refractive index was evaluated from the nonlinear phase change from "closed aperture" data and the nonlinear absorption coefficient from "open aperture" curves. The source used for these measurements consisted of $\approx 260 \mathrm{fs}$ pulses at $1.55 \mu \mathrm{m}$ generated by a Quantronix Palitra OPA pumped with a Ti: sapphire laser (Clark-MXR CPA 2001) at a repetition of 1 $\mathrm{kHz}$. The beam from the Palitra was truncated using an aperture to improve its spatial coherence and then focused onto the sample using a $125 \mathrm{~mm}$ focal length lens. The beam transmitted through the sample was intercepted in the far field by a rotating scattering screen (to reduce the speckle) and the light distribution on the screen was imaged onto a Xenics InGaAs camera. To ensure the accuracy of our z-scan method, we "calibrated" our measurements using an $\mathrm{As}_{2} \mathrm{~S}_{3}$ bulk glass sample as a reference since its nonlinearity is known accurately at $1550 \mathrm{~nm}$ from a wide range of experiments and has a value $n_{2}$ of $2.9 \pm 0.3 \times 10^{-14} \mathrm{~cm}^{2} / \mathrm{W}[5]$.

\section{Results and Discussion}

Figure 1 shows the measurement results of $T_{g}$ for the glasses. It is well established that, $T_{g}$ depends on the connectivity, and consequently on the rigidity of the vitreous network. ${ }^{10}$ As shown in Fig. $1, T_{g}$ of GeAsSe glasses exhibit almost linear behavior against mean coordination number(MCN), a parameter that is correlated with chemical composition of the glass, and is defined as the sum of the products of the individual abundance times the valence of the constituent atoms, irrespective of their actual chemical compositions. ${ }^{11}$ If we compare $\mathrm{T}_{\mathrm{g}}$ of the glasses with same MCN but different chemical compositions, we can see that the chemical compositions only induce a slight fluctuation from the linear behavior. From the structural viewpoint, it is well accepted that, for the glasses with low Ge content, Se chains along with Se rings dominate glass structures. With increasing Ge content, different molecular units including $\mathrm{AsSe}_{3 / 2}$ pyramids and $\mathrm{GeSe}_{4 / 2}$ tetrahedra will cross-link together until all Se atoms are consumed by $\mathrm{Ge}$ and As. Further addition of $\mathrm{Ge}$ content leads to the formation of the homopolar $\mathrm{Ge}-\mathrm{Ge}$ and As-As bonds in order to compensate the deficiency of Se. ${ }^{12,13}$ However, these homopolar Ge-Ge and As-As bonds have not destroyed the network connectivity, this might be due to the close position of these three elements in the periodic table and thus the replacement of one by another only induce negligible change in the structure and physical properties while forming the covalent network. This is the reason why we observed linear increase of $\mathrm{T}_{\mathrm{g}}$ in Fig.1.

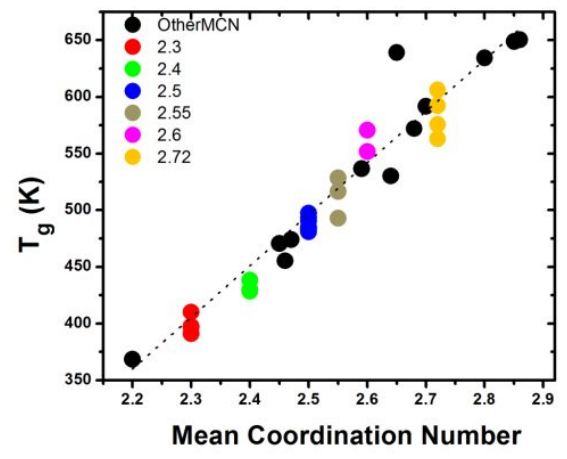

Fig.1. $\mathrm{T}_{\mathrm{g}}$ of the glasses as a function of $\mathrm{MCN}$.

Thermal parameters like activation energy of glass transition and fragility were further extracted from the DSC measurements with different scanning rates. The dependence of $T_{g}$ on the heating rate, $\alpha$, was found to obey the equation: ${ }^{10}$

$$
\mathrm{d} \ln \alpha / \mathrm{d}\left(1 / T_{g}\right)=-E_{a} / R
$$

where $E_{a}$ and $R$ are the activation energy for the glass transition and the ideal gas constant, respectively. The fragility index $m$ can be calculated from $T_{g}$ and $E_{a}$ using the relation: ${ }^{10}$ 


$$
m=E_{a} /\left(R T_{g} * \ln 10\right)
$$

where $E_{a}$ is the activation energy and $T_{g}$ is the glass transition temperature at $10 \mathrm{~K} / \mathrm{min}$. The fragility index characterizes the extent to which glass forming liquids depart from the Arrhenius relation of viscosity as they approach the glass transition. Generally it is expected that glasses with the lowest fragility index are the strongest glass formers. The variation of $E_{a}$ as a function of $\mathrm{MCN}$ is displayed in Fig. 2(a) and shows a sharp local minimum at $\mathrm{MCN}=2.40-2.45$. Data shown in Fig. 2(b), although scattered, exhibit a minimum around $\mathrm{MCN}=2.45-2.5$ where the strongest glass formers exist.
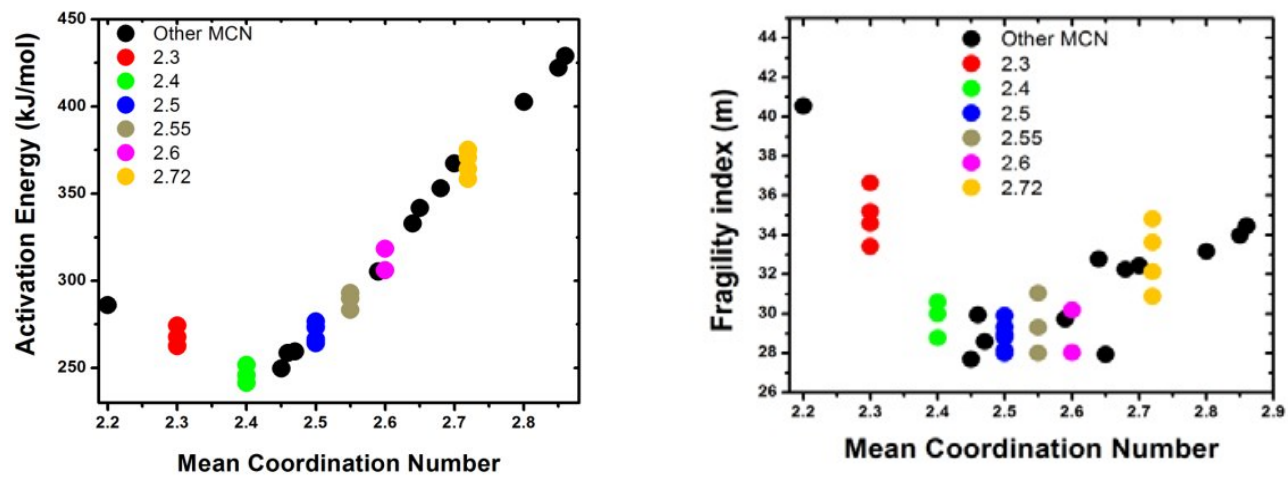

Fig.2. Glass transition activation energy (a) and fragility (b) as a function of MCN.

Let us move to the film-forming properties since the waveguide devices in mid infrared region are based on the chalcogenide films deposited on silicon wafers under vacuum where the materials are frozen in composition of vapour containing molecular clusters, etc. Film created under vacuum and thus non-equilibrium conditions generally results in different bonding structure from its bulk counterpart that is formed under thermodynamical equibrium conditions, and thus in turn leading to different physical properties ${ }^{14-18}$. Figure 3 (a) and (b) are refractive index and bandgap of the bulk glass and film, respectively. Most films created by thermal evaporation show different refractive index and optical bandgap compared with bulk glasses, and this indicates that films and bulks have different chemical bonds in most cases. However, it is evident that there is an exception around $\mathrm{MCN}=2.45$ where film and bulk properties are almost identical. This is in agreement with the results in Fig. 2 where the glass with a composition around MCN $=2.45$ is a strong glass former.
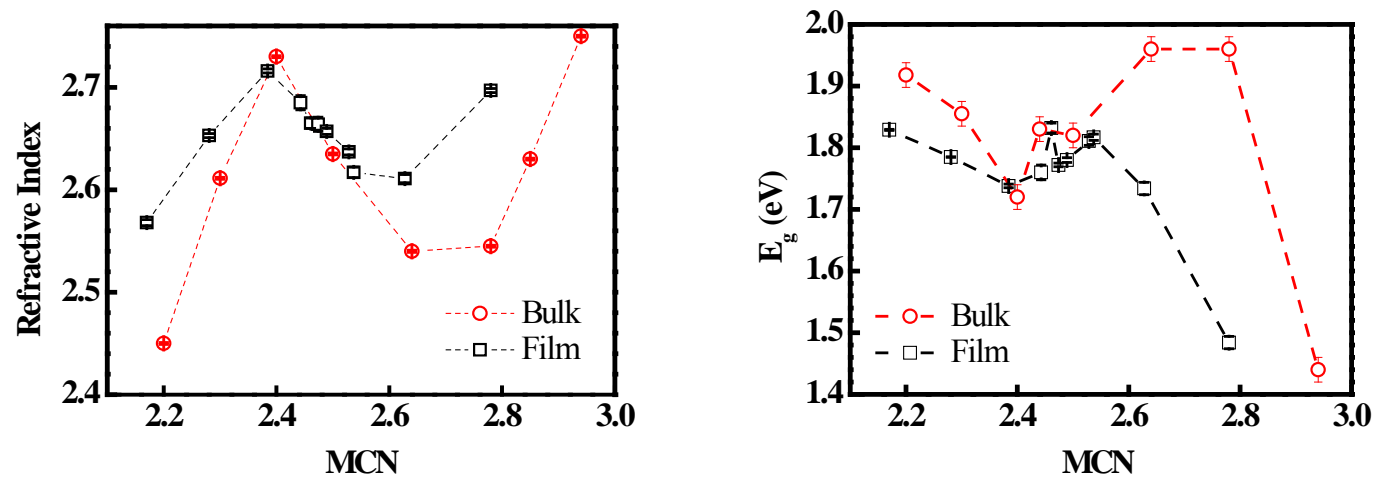

Fig.3. Refractive index (a) and optical bandgap (b) as a function of MCN.

We further performed the experiments to determine the photosensitivity of the films to band-edge light around 640nm. We irradiated the films with different compositions (and thus different $\mathrm{MCN}$ ) for 100h and measured the change of the refractive index and optical bandgap ${ }^{19}$. Figure 4 is the refractive index difference as a function of MCN. It was found that, high MCN films show large photosensitivity where the change of the refractive index could be 0.08 , while low $\mathrm{MCN}$ films have a small photosensitivity with a change of the refractive index of 0.03 . However, at around $\mathrm{MCN} \approx 2.45$, no detectable photosensitivity can be found in chalcogenide thin films. 


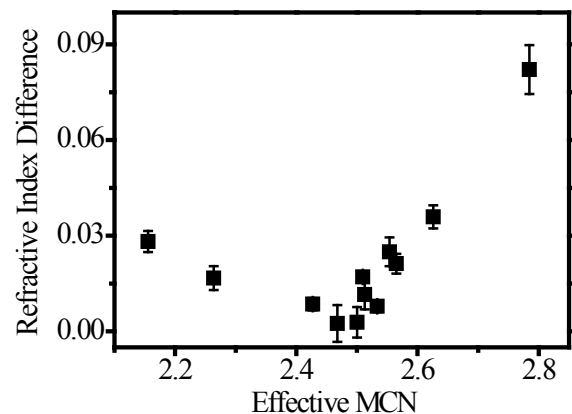

Figure 4. Refractive index difference as a function of MCN in Ge-As-Se films

Finally we measured the optical losses and linear and nonlinear refractive index of the GeAsSe glasses. As shown in Fig.5(a), it is clear that the optical loss of the glasses increases with increasing MCN. While the glasses with low MCN show low losses, the glasses with high MCN more than 2.7 have high losses which are unacceptable for the applications in photonics. Our recent Raman scattering measurements have indicated that the loss is correlated with the number of the defective bonds in the glasses ${ }^{7}$. Therefore, it is essential to choose the materials compositions with an MCN less than 2.7. Fig.5(b) shows the linear refractive index third order nonlinearity coefficiency of the bulk glasses as a function of MCN in groups of glasses containing $10 \%$ and $20 \%$ As, respectively. It can be found that, linear refractive index exhibits a minimum at around MCN 2.65, while nonlinear coefficiency shows a minimum at almost same position although the data are more scattered. Moreover, we note that the change of nonlinearity is from 5 to $8.7 \times 10^{-14} \mathrm{~cm}^{2} / \mathrm{W}$ where the fluctuation is less than $50 \%$.
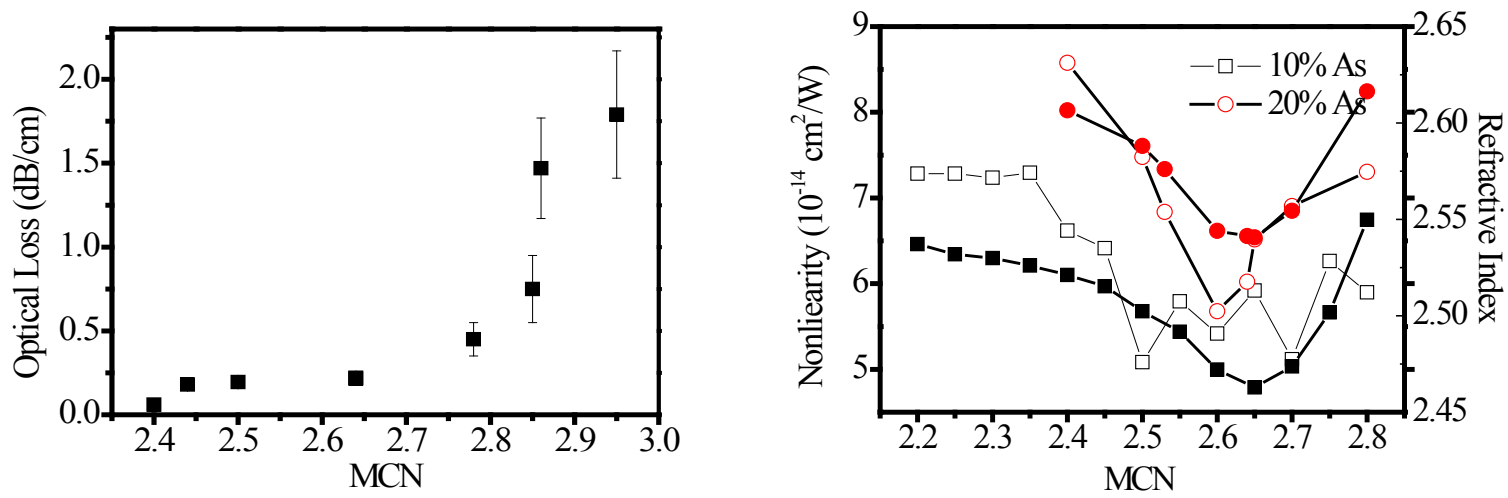

Figure 5. Optical loss of several GeAsSe glasses (a); and linear (solid) and nonlinear(open) refractive index of GeAsSe glasses as a function of $\mathrm{MCN}$.

For the best applications in photonics, basically the glasses should have relatively high $\mathrm{T}_{\mathrm{g}}$, high nonlinearity, low optical loss, high structural stability, and less photosensitivity. It is well known that, the structural relaxation is intrinsic in glass that always deteriorates the performance of glass-based photonics device. Therefore it is essential to seek a glass with strong glass-forming ability and thus less structural relaxation. Figure 2 (a) and (b) have clearly demonstrated that the strongest glass forming ability is located at a region with MCN around 2.45-2.5. The measurement of the physical properties indicated that, indeed, these glasses exhibit similar bonding structure to the films, and the properties are stable against any photo-induce changes. Since the glasses with MCNs more than 2.7 have larger optical loss, these glasses should be excluded in the use of photonics devices. Considering the structural stability and photosensitivity, the glasses with an MCN around 2.45-2.5 might be the best options for the application in photonics. The glasses at the region generally have a relative high $\mathrm{T}_{\mathrm{g}}$ of $450 \mathrm{~K}$, and the third order optical nonlinearity is around $6.5 \times 10^{-14} \mathrm{~cm}^{2} / \mathrm{W}$ that is 2 times than that in binary $\mathrm{As}_{2} \mathrm{~S}_{3}$ glass ${ }^{20}$. 


\section{Conclusion}

We prepared numbers of Ge-As-Se glasses and thin films, and measured various physical properties. We found that, the glasses with MCNs around 2.45-2.5 have strong glass-forming ability, and less photosensitivity, the glasses also exhibit relatively high $\mathrm{T}_{\mathrm{g}}$ and large optical nonlinearity of around $6.5 \times 10^{-14} \mathrm{~cm}^{2} / \mathrm{W}$. The glasses with the compositions in this region were considered the best for the applications in mid infrared wave guide devices.

\section{References}

[1] Wang, R., [Amorphous Chalcogenide], Pan Stanford Publishing, New York \& Boston, 199-248 (2014).

[2] Eggleton, B. J., Luther-Davies, B., and Richardson, K., "Chalcogeide Photonics", Nat. Photonics, 5, 141-148 (2011).

[3] Gai, X., Han,T., Prasad,A., Madden,S., Choi,D.Y., Wang,R.P., Bulla,D., and Luther-Davies, B.," Progress in Optical Waveguides Fabricated from Chalcogenide Glass", Opt. Express, 18, 26635-26646 (2010).

[4] Choi,D.Y., Madden,S., Bulla, D.A., Wang,R., Rode,A., and Luther-Davies,B., "Submicrometer-Thick Low-Loss $\mathrm{As}_{2} \mathrm{~S}_{3}$ Planar Waveguides for Nonlinear Optical Devices", IEEE Photonics Technology Letters 22, 495(2010).

[5] Lucas,P., Riley,M.R., Boussard-Pledel,C., and Bureau,B., "Advances in chalcogenide fiber evanescent wave biochemical sensing", Anal. Biochem. 351, 1-10 (2006).

[6] Choi,D.Y., Madden,S., Bulla,D., Wang,R., Rode,A., and Luther-Davies, B.,"Thermal annealing of arsenic trisulphide thin film and its influence on device performance", Journal of Applied Physics 107, 053106(2010).

[7] Prasad,A., Zha,C.J., Wang,R.P., Smith,A., Madden,S., and Luther-Davies, B., "Properties of $\mathrm{Ge}_{\mathrm{x}} \mathrm{As}_{\mathrm{y}} \mathrm{Se}_{1-\mathrm{x}-\mathrm{y}}$ glasses for all-optical signal processing”, Optics Express 16, 2804(2008).

[8] Choi,D.Y., Maden,S., Rode,A., Wang, R.P., Luther-Davies,B., "Plasma etching of $\mathrm{As}_{2} \mathrm{~S}_{3}$ films for optical waveguides", J. Non-Crystalline Solids 354, 3179(2008).

[9] Choi, D.Y., Madden,S., Rode,A., Wang,R.P., Luther-Davies,B., "Fabrication of Low loss $\mathrm{Ge}_{33} \mathrm{As}_{12} \mathrm{Se}_{55}$ Planar Waveguides", Applied Physics Letters 91, 011115(2007).

[10] Wang, T., Gulbiten, O., Wang,R.P., Yang,Z.Y.,Smith,A., Luther-Davies, B., and Lucas,P., "Relative contribution of stoichiometry and mean coordination towards the fragility of Ge-As-Se glass forming liquids", J.Phys.Chem.B 118, 1436-1442(2014).

[11] Wang,R.P., Bulla,D., Smith,A., and Luther-Davies, B., "Structure and Physical Properties of $\mathrm{Ge}_{\mathrm{x}} \mathrm{As}_{\mathrm{y}} \mathrm{Se}_{1-\mathrm{x}-\mathrm{y}}$ glasses with the same mean coordination number of 2.5", Journal of Applied Physics 109, 023517(2011).

[12] Wang, R.P., Smith, A., Luther-Davies,B., Kokkonen, H., Jackson, I., "Observation of two elastic thresholds in $\mathrm{Ge}_{\mathrm{x}} \mathrm{As}_{\mathrm{y}} \mathrm{Se}_{1-\mathrm{x}-\mathrm{y}}$ glasses", Journal of Applied Physics 105, 056109 (2009).

[13] Opletal, G., Wang, R.P., Russo,S.P., "Bonding trends within ternary isocoordinate chalcogenide glasses $\mathrm{Ge}_{\mathrm{x}} \mathrm{As}_{\mathrm{y}} \mathrm{Se}_{1-}$ x-y", Physical Chemistry Chemical Physics 15, 4582-4588 (2013).

[14] Bulla,D. Wang,R.P., Prasad,A., Rode,A.V., Madden,S.J., and Luther-Davies,B., "On the properties and stability of thermally evaporated Ge-As-Se thin films", Applied Physics A: Material Sciences and Processing 96, 615(2009).

[15] Wang,R.P., Rode,A.V., Zha,C.J., Madden,S.J., and Luther-Davies, B., "Annealing induced phase transformation in amorphous $\mathrm{As}_{2} \mathrm{~S}_{3}$ films", Journal of Applied Physics 100, 063524(2006).

[16] Wang,R.P.,Choi,D.Y.,Rode,A.V.,Madden,S.J., and Luther-Davies, B., "Rebonding of Se to As and Ge in $\mathrm{Ge}_{33} \mathrm{As}_{12} \mathrm{Se}_{55}$ films upon thermal annealing: evidence from XPS investigations", Journal of Applied Physics 101, 113517(2007).

[17] Javis,R.A., Wang,R.P., Rode,A.V., Zha,C.J., Krolikowska, M., Madden,S.J., Choi,D.Y., Kolev,V.Z., and LutherDavies,B., "Thin film deposition of $\mathrm{Ge}_{33} \mathrm{As}_{12} \mathrm{Se}_{55}$ by pulsed laser deposition and thermal evaporation: comparison of properties", Journal of Non-crystalline Solids 353, 947-949(2007).

[18] Wang, R.P., Rode, A.V., Madden,S.J., Zha,C.J., Javis, R.A., and Luther-Davies, B., "Structural relaxation and optical properties in amorphous $\mathrm{Ge}_{33} \mathrm{As}_{12} \mathrm{Se}_{55}$ films", Journal of Non-crystalline Solids 353, 950-952(2007).

[19] Su, X.Q., Wang, R.P., and Luther-Davies, B., "The dependence of photosensitivity on composition for thin films of $\mathrm{Ge}_{\mathrm{x}} \mathrm{As}_{\mathrm{y}} \mathrm{Se}_{1-\mathrm{x}-\mathrm{y}}$ chalcogenide glasses", Applied Physics A 113, 575-581(2013).

[20] Wang,T.,Gai,X.,Wei,W., Wang, R., Yang, Z., Xiang,S., Madden, S., and Luther-Davies, B., "Systematic Z-scan measurement for the nonlinearity of chalcogenide glasses", Optical Materials Express 4, 1011-1022(2014). 